\title{
Exploration of the optimal skin-camera distance for facial photoplethysmographic imaging measurement using cameras of different types
}

\author{
Bin Han \\ Shenzhen Institutes of Advanced \\ Technology, Chinese Academy of \\ Sciences \\ Xueyuan Avenue 1068, Shenzhen \\ 518055, P.R. China. \\ (+86)18655178325 \\ bin.han@siat.ac.cn
}

\author{
Kamen Ivanov, Yan Yan \\ Shenzhen Institutes of Advanced \\ Technology, Chinese Academy of \\ Sciences \\ Xueyuan Avenue 1068, Shenzhen \\ 518055, P.R. China. \\ $(+86) 18898561517$ \\ kamen@siat.ac.cn
}

\author{
Lei Wang* \\ Shenzhen Institutes of Advanced \\ Technology, Chinese Academy of \\ Sciences \\ Xueyuan Avenue 1068, Shenzhen \\ 518055, P.R. China. \\ $(+86) 15818518450$ \\ wang.lei@siat.ac.cn
}

\begin{abstract}
Recent studies have provided strong evidence that photoplethysmographic imaging (PPGi) techniques can serve for measuring the heart rate from video recordings. However, in the works on PPGi technology so far, motion artifacts, which limit the accuracy, were unavoidable. In this work, we present an exploration of our assumption that for a particular model of camera, there is an optimal measurement distance, which ensures the minimal influence of artifacts in PPGi measurement.We conducted experiments using two cameras of different types that are commonly integrated with modern consumer electronic devices. First, we demonstrated that the both types of cameras are applicable for PPGi-based non-contact measurement. We used the both cameras simultaneously to record the face regions of 10 subjects and then extracted the information about their heart rates from each of the recordings. To verify the results obtained by PPGi method, we compared them with those measured using a "gold standard" technique. We then explored the relation between the camera-face distance and the measurement error. For each kind of camera, we determined the optimal face-camera distance that ensured reducing the error from motion artifacts to the possible minimum.
\end{abstract}

\section{Categories and Subject Descriptors}

J.3 [Computer Applications]: Life and medical sciences Health, medical information systems.

\section{General Terms}

Measurement, Performance, Experimentation, Human Factors.

\section{Keywords}

Heart Rate, Photoplethysmographic imaging, optimal distance estimation.

\section{INTRODUCTION}

The regular and non-invasive assessment of the cardiovascular function is of great importance for the prevention of cardiovascular problems and the treatment of chronic cardiovascular diseases [1]. Resting heart rate is the simplest parameter that can serve as an independent indicator of the risk of occurrence of a cardiovascular disease [2]. Until the present moment, the "gold standard" techniques that allow measuring the heart rate require the electrodes or sensors to be in direct contact with the skin, which costs the comfort of the patient. Such is the case, for example, with the conventional electrocardiographic equipment. In contrast, a non-contact heart rate measurement method, based on portable multi-functional consumer devices could allow enormous benefits for the patient.

One potential way for convenient non-contact heart rate measurement is to adopt photoplethysmographic imaging (PPGi) techniques. Because of the pulsatile nature of the human body circulatory system, photoplethysmographic techniques can be used for measuring the variations of blood volume [3, 4]. The idea of performing physiological measurements using signals captured from the face was introduced by Pavlidis [5]. Further, a strict proof of using measurements from the face has been given through analysis of facial thermal videos $[6,7]$. These techniques allow to measure the heart generated pulse wave in a non-contact manner. Later, Poh et al. reported a web camera-based technique for non-contact acquisition of PPGi signals that used ambient light as a source of illumination [8]. Daniel McDuff et al. measured the blood volume pulse using PPGi signals obtained using a five-band digital cameras remotely. Thus, the combination of cyan, green, and orange bands allowed for a better result compared to the case of using the green band only $[9,10]$. All these works demonstrate the high potential of noncontact methods for measurement of human body physiological parameters. Modern smartphones usually have at least one highresolution camera and offer high computational speeds. Taking into account their small form factor, they could serve as portable measurement stations. Therefore, the research of PPGi measurement methods using a smartphone camera is of great significance.

A major problem of the camera based non-contact physiological information measurement is the presence of motion artifacts [19]. When capturing the face region, motion artifacts are determined 
by involuntary head motions including the Newtonian reaction to the influx of blood at each beat $[8,17,18]$. Another part of the error arises from the inter-trial variability in the selection of the region of interest (ROI), that is usually associated with the utilization of a face tracker. We hypothesized that the influence of motion artifacts could be reduced by proper adjustment of the measurement distance. If camera-specific information about the optimal measurement distance is available to an automated measurement application, it could allow for fast adjusting of the user face position.

With the present paper, we aim to demonstrate the influence of the measurement distance on the error when using PPGi technology for heart rate measurement. For this goal, we obtained the heart rate value from a sequence of video images using two different kinds of cameras. We compared the results obtained using each type of camera with those, obtained using a "gold standard" measurement technique and drew conclusions about the measurement accuracy. To our best effort, we did not find other exploration of the relation between the face-camera distance and the measurement accuracy in the existing research literature dedicated to PPGi methods.

\section{METHODS}

\subsection{Experimental Procedure}

We recruited ten healthy subjects from the Shenzhen Institutes of Advanced Technology, Chinese Academy of Sciences. In Table 1 we provide data about the gender, age, average heart rate, and ambient temperature during the recording for each subject. We conducted all experiments indoors under a varying amount of ambient sunlight entering through the windows as the only source of illumination. For recording of videos, we used a builtin laptop camera and a smartphone camera. The model of the built-in laptop web camera was ThinkPad T440p by Lenovo Inc. The smartphone camera was the built-in one of iPhone $4 \mathrm{~s}$ by Apple Inc. In all experiments, we used a PPG module of type TPTSD200A from BIOPAC to acquire the pulse rate from the right fingertip in the role of a "gold standard". Fig. 1 illustrates the experimental setup. Prior to collecting the data, we asked each participant to sit still, breathe spontaneously, and look at the cameras during the video recording. For each subject, we recorded data for face-camera distances of $0.3 \mathrm{~m}, 0.6 \mathrm{~m}, 0.9 \mathrm{~m}$, $1.2 \mathrm{~m}, 1.5 \mathrm{~m}$ and $1.8 \mathrm{~m}$. At each distance, we recorded data with duration of $30 \mathrm{~s}$, simultaneously from the both cameras. We ensured the proper focusing to obtain clear images. Captured videos were with a resolution of $640 \times 480$ pixels. The only supported frame rate of the both cameras is 30 frames per second. We recorded the videos from the both cameras in color, i.e. we obtained signals from the R, G and B color channels. Each channel of each camera was sampled with a resolution of 8 bits.

\subsection{Recovery of Heart Rate}

We analyzed all the video and physiological recordings offline using our custom software under MATLAB, following the steps illustrated in Fig. 2. First, we used an automated face tracker to localize the face region within each video frame. For this goal, we utilized a freely available tracking algorithm based on the work of Kaihua Zhang [14]. In contrast to commonly used algorithms such as $[20,21]$, it ensures high repeatability of the ROI selection. Thus, in the experiment, we ensured that the error induced by the face tracker will be negligible. A feature that
Table 1.Data for subject during the experiment: gender, age, heart rate, and ambient temperature

\begin{tabular}{|c|c|c|c|c|}
\hline Subj. No & Gender & Age & $\mathrm{HR}(\mathrm{bpm})$ & Temp. ${ }^{0} \mathrm{C}$ \\
\hline 1 & $\mathrm{M}$ & 24 & 97 & 24.6 \\
\hline 2 & $\mathrm{~F}$ & 22 & 72 & 24.4 \\
\hline 3 & M & 25 & 70 & 24.5 \\
\hline 4 & M & 31 & 61 & 24.6 \\
\hline 5 & M & 24 & 73 & 24.5 \\
\hline 6 & $\mathrm{~F}$ & 26 & 78 & 24.8 \\
\hline 7 & M & 29 & 84 & 24.6 \\
\hline 8 & M & 42 & 89 & 24.6 \\
\hline 9 & M & 34 & 76 & 24.7 \\
\hline 10 & $\mathrm{~F}$ & 24 & 93 & 24.5 \\
\hline & $\begin{array}{l}\text { classical } \\
\text { asureme } \\
\text { gold sta }\end{array}$ & aptop & in camera & \\
\hline
\end{tabular}

Fig 1. Experimental setup

additionally contributes to a small error is that this algorithm can track arbitrarily selected skin regions. The ROI of each frame was then separated into R, G, and B channels. For each frame, we averaged the ROI pixel values to yield a single value. The obtained values for each channel formed its raw PPGi signal. To reduce the influence of the motion artifacts, we adopted the method of independent component analysis (ICA). Before passing the three signals to ICA, they were normalized. The details on the application of ICA for our particular case are explained in [8]. From ICA, we obtained three independent component signals. By visual observation, we determined the one of them that contains the heart rate signal. We then used a fifthorder Butterworth band-pass filter on it, setting a passband that corresponds to a relatively wide heart rate range from $0.75 \mathrm{~Hz}$ to $3 \mathrm{~Hz}$ (i.e. heart rates from $45 \mathrm{bpm}$ to $180 \mathrm{bpm}$ ). We then applied a moving average smoothing on the filtered signal. Finally, we applied fast Fourier transform on the filtered and smoothened signal.

\section{RESULTS}

\subsection{Heart Rate Signal Recovering}

Fig. 3 shows the results for one of the participants. The cardiovascular pulse waves in the recovered independent source signal are visible, and the heart rate measured using the PPGi technique corresponds to the one measured with the reference system. To assess the agreement between results obtained from the both kinds of techniques for all the subjects, we performed a Bland-Altman analysis [15] (Fig. 4a,c). We used $\alpha$ (type I error) set at 0.05 . In Fig. 4 b,d we also provide scatter plots. In Fig. 4a, the mean bias between $\mathrm{HR}_{\text {gold }}$ and $\mathrm{HR}_{\text {webcam }}$ is $0.40 \mathrm{bpm}$ with 95\% limits of agreement from $-3.32 \mathrm{bpm}$ to $4.12 \mathrm{bpm}$. In Fig. 4c the mean bias between $\mathrm{HR}_{\text {webcam }}$ and $\mathrm{HR}_{\text {smartphone }}$ is $0 \mathrm{bpm}$ with the corresponding $95 \%$ limits of agreement from -2.92 to 2.92 bpm. We found a significant correlation between the PPGi signals obtained from the web camera and smartphone camera, respectively, with $r^{2}>0.8$ and $p<0.01$ for all correlations. We 
performed the Bland-Altman analysis and the scatter test using SPSS v19.0.

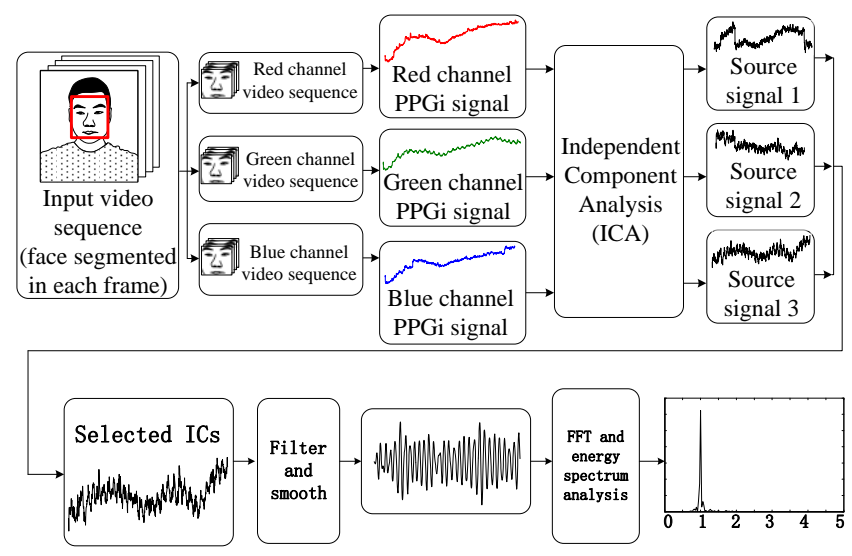

Fig 2. The automated method we applied to recover the cardiac pulse.

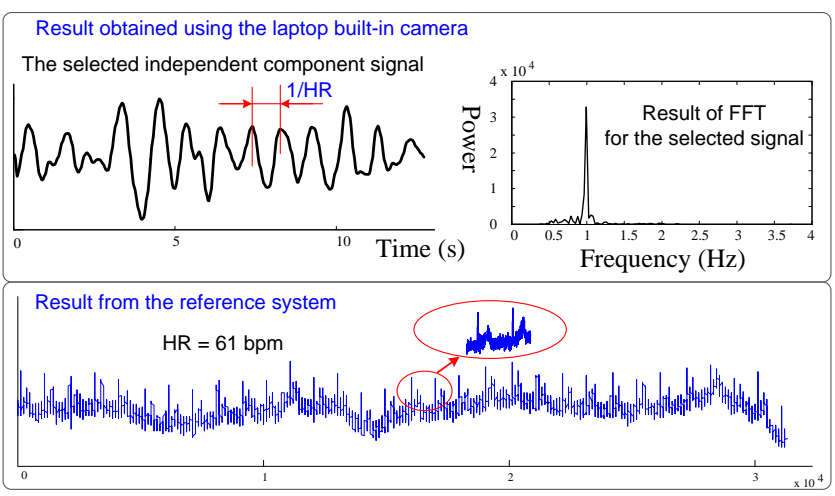

Fig 3. Obtained cardiac pulse of one participant.

\subsection{Face-Camera Distance and Accuracy}

For different measurement distances we obtained a different heart rate measurement result. The error between the results obtained from the reference system and the PPGi system is defined as:

$$
\varepsilon=\frac{|60 f-M|}{M}
$$

where $f$ is the pulse frequency measured using PPGi technique and $M$ represents the heart rate measured by the reference system. For the ten tested subjects, we obtained a similar behavior of the relation between the measurement distance and the associated error. In Table 2 we provide a representative sample for two of the participants. We provide a more intuitive, graphical representation of the data from Table 2 in Fig. 5. In Fig. 5 we also show a fitting curve, an approximation of the "errordistance" relation. It could be used to build a model for obtaining the optimal estimation.

\section{DISCUSSION}

In this study, we explore how the measurement distance affects the quality of heart rate photoplethysmographic measurement when using a smartphone camera and a webcam under ambient light. To achieve the goal, we ensured to have no sources of significant error but the motion artifacts provoked from involuntary head motions. For example, a typical problem in real-time video processing is the "losing" of frames when the time for processing exceeds the time between two subsequent frames. In our case, we did not face this problem since we processed all data offline. Our choice of a face tracker, and the fact that each

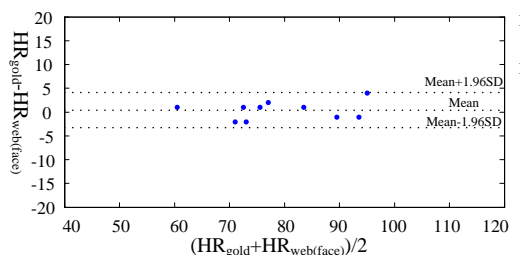

(a)

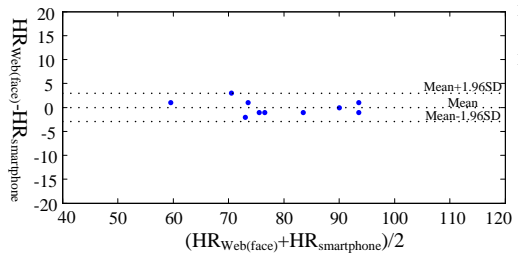

(c)

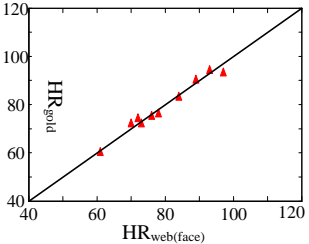

(b)

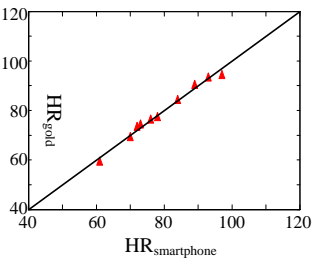

(d)
Fig 4. Comparison of measurements obtained from the both kinds of cameras for all the ten subjects. (a,c) Bland-Altman plots. (b,d) Scatter plots

Table 2. Distance vs. accuracy for two of the subjects

\begin{tabular}{ccccccc}
\hline Subj.1 & Dist. & HR gold $_{\text {gaptop }}$ & Error & Phone & Error \\
\hline & 0.3 & 97 & 1.46 & 0.0969 & 1.48 & 0.0845 \\
& 0.6 & 97 & 1.50 & 0.0722 & 1.51 & 0.0660 \\
& 0.9 & 97 & 1.58 & 0.0227 & 1.56 & 0.0351 \\
& 1.2 & 97 & 1.55 & 0.0412 & 1.55 & 0.0412 \\
& 1.5 & 97 & 1.50 & 0.0722 & 1.51 & 0.0660 \\
& 1.8 & 97 & 1.48 & 0.0845 & 1.47 & 0.0907 \\
\hline Subj.2 & Dist. & HR gold $_{\text {gld }}$ & Laptop & Error & Phone & Error \\
\hline & 0.3 & 61 & 0.94 & 0.0754 & 1.95 & 0.0656 \\
& 0.6 & 61 & 0.97 & 0.0459 & 1.98 & 0.0361 \\
& 0.9 & 61 & 1.01 & 0.0066 & 1.02 & 0.0033 \\
& 1.2 & 61 & 1.03 & 0.0131 & 1.03 & 0.0131 \\
& 1.5 & 61 & 1.06 & 0.0426 & 1.06 & 0.0426 \\
& 1.8 & 61 & 1.08 & 0.0623 & 1.09 & 0.0721 \\
\hline
\end{tabular}

subject was asked to do his best to keep his head still, allowed to avoid a significant error from the face tracker processing. Table 3 shows the results from the best measurement of each subject and each camera. For the two kinds of cameras, the best results were obtained in the same trials. The results obtained by the PPGi technique showed a very high agreement with the reference contact measurements. Based on Fig. 4, we also confirmed that both the web- and the smartphone- camera based PPGi systems allowed for successful measurement of the heart rate in all the tested subjects. We found that when the distance was too short, the error from motion artifacts became significant. On the other hand, when the distance was too large, it was difficult to detect the cardiac pulse. The latter case is assotiated with a low amplitude of the useful signal frequency component in the power 
spectrum. Therefore, the distance between the face and the camera is a significant factor, which affects the PPGi measurement accuracy. The optimal distance for PPGi measurement needs to be determined for each particular type of camera. By analyzing the fitting curves for the two kinds of cameras and all the tested subjects, we found that the optimal measurement distance fell in the range from $0.9 \mathrm{~m}$ to $1.2 \mathrm{~m}$ (the lowest points of the curves in Fig. 5).
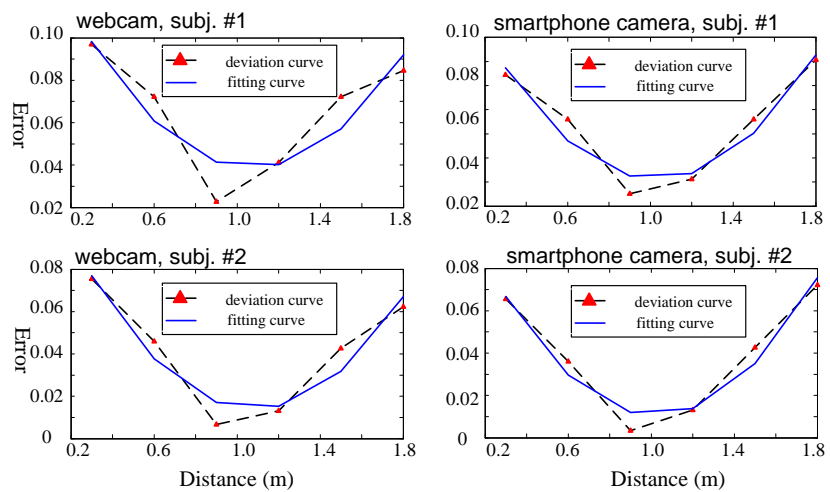

Fig 5. PPGi heart rate measurement error vs. face-camera distance

Table 3. Best measurement for each subject and each camera

\begin{tabular}{cccc}
\hline Subj. & HR $_{\text {gold }}$ & HR $_{\text {PPGi-laptop }}$ (error) & HR $_{\text {PPGi-phone }}$ (error) \\
\hline 1 & 97 & $93(0.041)$ & $94(0.031)^{*}$ \\
2 & 72 & $74(0.028)$ & $73(0.014)^{*}$ \\
3 & 70 & $72(0.028)$ & $69(0.014)^{*}$ \\
4 & 61 & $60(0.016)^{*}$ & $59(0.033)$ \\
5 & 73 & $72(0.014)$ & $74(0.014)$ \\
6 & 78 & $76(0.026)$ & $77(0.013)^{*}$ \\
7 & 84 & $83(0.012)$ & $84(0.000)^{*}$ \\
8 & 89 & $90(0.011)$ & $90(0.011)$ \\
9 & 76 & $75(0.013)$ & $76(0.000)^{*}$ \\
10 & 93 & $94(0.011)$ & $93(0.000)^{*}$ \\
\hline \multicolumn{4}{c}{ "**" represents the minimum error } \\
\hline
\end{tabular}

\section{CONCLUSION}

With this study, we demonstrated that cameras of two different kinds both served well in the application of PPGi measurement of heart rate. The both tested cameras were incorporated in portable consumer devices. Thus, we confirmed that there is a base for the development of human physiological parameter monitoring applications, based on consumer devices such as mobile phones and laptops. However, when developing such applications, to ensure highly accurate measurements, the properties of each particular camera should be taken into account. Moreover, the measurement can be facilitated by using approximation models that express the relation between the error and measurement distance. The limitation of our study is that we obtained results for two particular models of cameras, while we did not consider the influence of parameters that will allow us to generalize our findings. Therefore, in our future work we intend to explore the measurement distance influence while taking more factors into account, aiming at providing of generalized results. We will also investigate the distance-error models in more detail, allowing for their application in automated measuring applications.

\section{ACKNOWLEDGMENTS}

This work was supported in part by the National 863 Program of China under Grant 2012AA02A604, in part by the Guangdong Innovation Research Team Funds for Low-Cost Healthcare and Image-Guided Therapy, in part by the Shenzhen Science and Technology Innovation Project under Grant CXZZ2013051710 4329683, and in part by the Next Generation Communication Technology-Major Project of National Scientific and Technological Programs under Grant 2013ZX03005013.

\section{REFERENCES}

[1] Cook, Stéphane, et al. "High heart rate: a cardiovascular risk factor?." European heart journal 27.20 (2006): 2387-2393.

[2] Fagundes, Jaqueline Eilert, and Iran Castro. "Predictive value of resting heart rate for cardiovascular and all-cause mortality." Arquivos brasileiros de cardiologia 95.6 (2010): 713-719.

[3] Hertzman, Alrick B. "Photoelectric plethysmography of the fingers and toes in man." Experimental Biology and Medicine 37.3 (1937): 529-534.

[4] Allen, John. "Photoplethysmography and its application in clinical physiological measurement." Physiological measurement 28.3 (2007): R1.

[5] Pavlidis, Ioannis, et al. "Interacting with human physiology." Computer Vision and Image Understanding 108.1 (2007): 150-170.

[6] Garbey, Marc, et al. "Contact-free measurement of cardiac pulse based on the analysis of thermal imagery." Biomedical Engineering, IEEE Transactions on 54.8 (2007): 1418-1426.

[7] Fei, Jin, and Ioannis Pavlidis. "Thermistor at a distance: unobtrusive measurement of breathing." Biomedical Engineering, IEEE Transactions on 57.4 (2010): 988-998.

[8] Poh, Ming-Zher, Daniel J. McDuff, and Rosalind W. Picard. "Noncontact, automated cardiac pulse measurements using video imaging and blind source separation." Optics express 18.10 (2010): 10762-10774.

[9] McDuff, Daniel, Sarah Gontarek, and Rosalind W. Picard. "Improvements in remote cardio-pulmonary measurement using a five band digital camera." (2014): 1-1.

[10] McDuff, Daniel, Sarah Gontarek, and Rosalind W. Picard. "Remote Detection of Photoplethysmographic Systolic-Diastolic Peaks Using a Digital Camera." (2014).

[11] Comon, Pierre. "Independent component analysis, a new concept?." Signal processing 36.3 (1994): 287-314.

[12] James, Christopher J., and Christian W. Hesse. "Independent component analysis for biomedical signals." Physiological measurement 26.1 (2005): R15.

[13] Zijlstra, W. G., A. Buursma, and W. P. Meeuwsen-Van der Roest. "Absorption spectra of human fetal and adult oxyhemoglobin, deoxyhemoglobin, carboxyhemoglobin, and methemoglobin." Clinical chemistry 37.9 (1991): 1633-1638.

[14] Zhang Kaihua, Lei Zhang, and Ming-Hsuan Yang. "Real-time compressive tracking." Computer Vision-ECCV 2012. Springer Berlin Heidelberg, 2012. 864-877.

[15] Bland, J. Martin, and DouglasG Altman. "Statistical methods for assessing agreement between two methods of clinical measurement." The lancet 327.8476 (1986): 307-310.

[16] Verkruysse, Wim, Lars O. Svaasand, and J. Stuart Nelson. "Remote plethysmographic imaging using ambient light." Optics express 16.26 (2008): 21434-21445.

[17] $\mathrm{Wu}, \mathrm{Hao}-\mathrm{Yu}$, et al. "Eulerian video magnification for revealing subtle changes in the world." ACM Trans. Graph. 31.4 (2012): 65.

[18] Balakrishnan, Guha, Fredo Durand, and John Guttag. "Detecting pulse from head motions in video." Computer Vision and Pattern Recognition (CVPR), 2013 IEEE Conference on. IEEE, 2013.

[19] Rhee, Sokwoo, Boo-Ho Yang, and Haruhiko Harry Asada. "Artifactresistant power-efficient design of finger-ring plethysmographic sensors." Biomedical Engineering, IEEE Transactions on 48.7 (2001): 795-805.

[20] Viola P, Jones M. Rapid object detection using a boosted cascade of simple features[C]//Computer Vision and Pattern Recognition, 2001. CVPR 2001. Proceedings of the 2001 IEEE Computer Society Conference on. IEEE, 2001, 1: I-511-I-518 vol. 1.

[21] Lienhart R, Maydt J. An extended set of haar-like features for rapid object detection[C]//mage Processing. 2002. Proceedings. 2002 International Conference on. IEEE, 2002, 1: I-900-I-903 vol. 1 